\title{
Delayed MRI Enhancement of Colorectal Cancer Liver Metastases Is Associated With Metastatic Mutational Profile
}

\author{
ARUN SETH ${ }^{1,2}$, YUTAKA AMEMIYA ${ }^{2}$, HELEN CHEUNG ${ }^{3}$, \\ EUGENE HSIEH $^{1}$, CALVIN LAW $^{4}$ and LAURENT MILOT ${ }^{3}$ \\ ${ }^{1}$ Department of Laboratory Medicine and Molecular Diagnostics, Sunnybrook Health Sciences Centre, \\ Department of Laboratory Medicine and Pathobiology, University of Toronto, Toronto, ON, Canada; \\ ${ }^{2}$ Genomics Core, Sunnybrook Research Institute, Sunnybrook Health Sciences Centre, Toronto, ON, Canada; \\ ${ }^{3}$ Department of Medical Imaging, Sunnybrook Health Sciences Centre, University of Toronto, Toronto, ON, Canada; \\ ${ }^{4}$ Department of Surgical Oncology, Odette Cancer Centre, \\ Sunnybrook Health Sciences Centre, University of Toronto, Toronto, ON, Canada
}

\begin{abstract}
Background/Aim: Individual tumor genomics plays a key role in determining patient prognosis, response to chemotherapy and in guiding therapy. In prior studies, it was shown that the degree of late enhancement of colorectal liver metastases (CRCLM) target tumor enhancement (TTE) as seen on magnetic resonance imaging (MRI) was associated with overall survival. In order to better understand the relationship between MRI enhancement and survival, the aim of this study was to characterize genomic profiles of tumors clustered by MRI TTE, and investigate the association between TTE and genetic mutations. Materials and Methods: Matched tumor and normal tissue samples from patients with weak TTE and strong TTE were analyzed by Next-generation sequencing (NGS) technology using a custom colorectal cancer panel. Results: We discovered a total of 42 non-synonymous somatic mutations from 10 patients with weak TTE and 26 with 10 patients with strong TTE. Adenomatosis Polyposis Coli (APC) was the most commonly altered gene, 18 of those APC mutations were found in the weak TTE and 9 in the strong TTE group. Conclusion: An association exists between TTE and mutational status of CRCLM, which may offer some explanation as to why TTE is associated with overall survival in patients with CRCLM.
\end{abstract}

This article is freely accessible online.

Correspondence to: Arun Seth, Ph.D., Biological Sciences Platform, Sunnybrook Institute, Room S238, 2075 Bayview Ave, Toronto, ON M4N 3M5, Canada. Tel: +1 4164806100 extension 3536, Fax: +1 4164805737, e-mail: arun.seth@utoronto.ca

Key Words: Colorectal cancer, liver metastasis, gene mutations, magnetic resonance imaging (MRI).
Colorectal cancer (CRC) is the 2nd leading cause of cancerrelated deaths in North America $(1,2)$. Most deaths are related to metastatic disease, usually to the liver. Despite developments in new treatment techniques, and especially in surgery associated with neoadjuvant or adjuvant chemotherapy, there remains a large range of survival and response to standard treatments between different individual patients, likely due to the "biology" (or subtypes) of individual cancers (3).

There is a growing body of evidence that individual tumor biology that can be distinguished through histology and genetic signatures is playing a key role in determining patient prognosis, response to chemotherapy and in guiding therapy (4-9).

Incorporating these markers into clinical practice is limited by the requirement of direct tissue sampling of the metastases within the liver, which is invasive, costly, and prone to under-sampling (10). Furthermore, tissue characterization is mostly determined via histology of the primary cancer which does not adequately extrapolate to metastatic disease that is by nature heterogeneous and susceptible to mutation over time (11-15).

An ideal prognostic biomarker would provide metastasesspecific characterization using non-invasive techniques amenable to repeated measurement and be widely available in Centers that manage colorectal cancer. It would both help choose the patients best suited to undergo surgery and help optimize their pre- and peri-operative treatment and guide chemotherapy in inoperable patients. Magnetic resonance imaging (MRI), is a non-invasive imaging method that is generally accepted as the best imaging method for diagnosis and staging of colorectal liver metastases (CRCLM) $(16,17)$.

In a prior study, it was shown that the degree of enhancement of the CRCLM, the MRI target tumor enhancement (TTE), were associated with overall survival following liver surgery $(18,19)$. At 3 years, $88.4 \%$ of patients with high TTE on the 


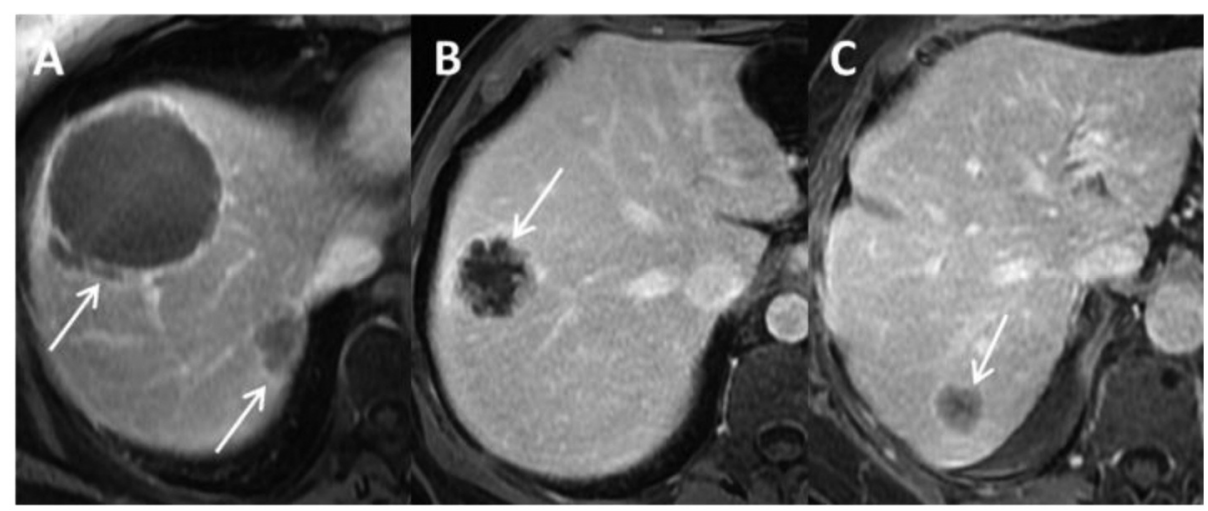

Weak TTE cases

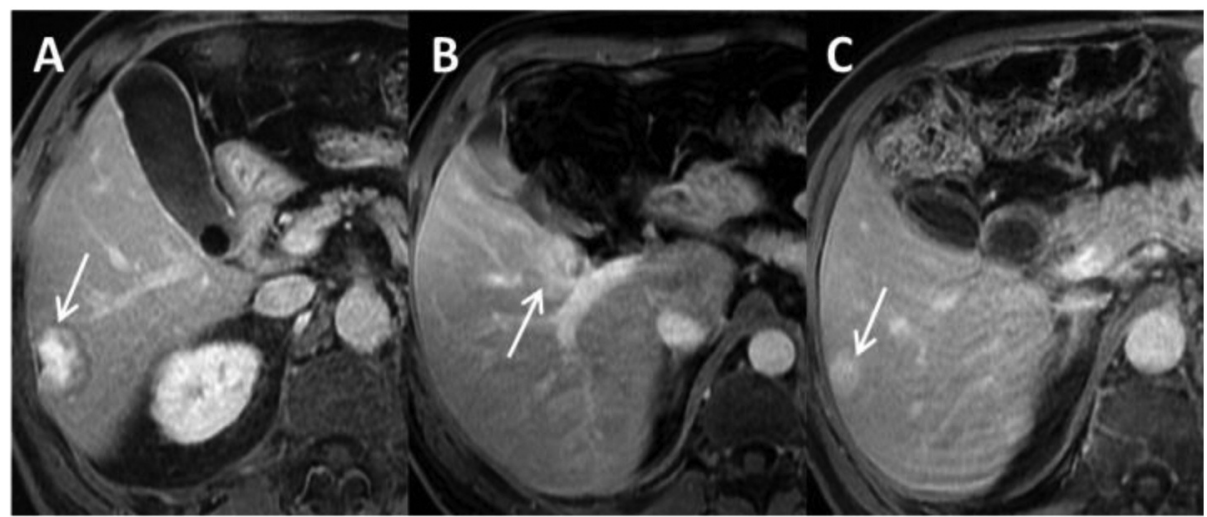

Strong TTE cases

Figure 1. Three examples of patients with colorectal cancer liver metastases (white arrows) demonstrating weak (top panel) and strong (bottom panel) target tumour enhancement (TTE) on 10-minute delayed phase magnetic resonance imaging (MRI) with gadobutrol.

preoperative MRI were alive vs. $58.8 \%$ in patients with low TTE. TTE was an independent marker of survival in Mutivariate Cox-Regression statistics. In this study, the association between TTE and histology was estimated, with a positive association being shown between TTE and fibrosis and a negative association being seen between TTE and necrosis. However, the potential association between TTE and tumor mutation was not investigated. Accordingly, the purpose of this study was to perform next-generation deep sequencing of tumors clustered by TTE, and investigate the association between TTE and mutations.

\section{Materials and Methods}

This retrospective study was approved by the Institutional Review Board and need for informed consent was waived.

Specimens. The patients from this study were part of a retrospective cohort, which included all patients at a single tertiary cancer center with CRCLM who had received a gadobutrol-enhanced MRI after treatment with chemotherapy (variable regimens as determined by standard of care, clinical treatment) and prior to hepatic resection for curative intent between January 1, 2006, and December 31, 2012. The details of the cohort and calculation of TTE were described previously (19).

Tumors were clustered based on their individual TTE. Matched tumor and adjacent normal tissue samples from 10 patients with weak TTE (contrast-to-noise ratio (CNR): -111.2 to -10.8 ) and 10 patients with strong TTE (CNR: 15.9 to 74.0) were selected from the cohort for the genomic analysis (Figure 1 and Table I). The choice of the tumor was performed based on TTE only and blinded from clinical information, survival and histopathologic analyses.

Genomic DNA extraction. Four $5 \mu \mathrm{m}$ sections were cut from the formalin-fixed, paraffin-embedded (FFPE) tissue block of colorectal cancer liver metastases and normal liver from post-hepatectomy pathology specimens. One section was Hematoxylin and Eosin (H\&E) stained and area of tumor was marked under microscope. This $\mathrm{H} \& \mathrm{E}$ slide was used as a guide slide for genomic DNA (gDNA) extraction from unstained sections. The gDNA was extracted by the 
Table I. List of Study ID, contrast-to-noise ratio (CNR) of the target lesion and Magnetic resonance imaging (MRI) phenotype (weak versus strong target tumor enhancement) used in this study.

\begin{tabular}{lrc}
\hline Study ID & Lesion (CNR) & MRI Category \\
\hline 249 & $-111,1951$ & Weak \\
15 & $-104,7803$ & Weak \\
659 & $-46,9107$ & Weak \\
292 & $-40,4455$ & Weak \\
568 & $-47,8899$ & Weak \\
643 & $-35,8564$ & Weak \\
391 & $-67,0529$ & Weak \\
778 & $-28,7722$ & Weak \\
288 & $-10,531$ & Weak \\
314 & $-10,8065$ & Weak \\
651 & 15,9208 & Strong \\
531 & 19,4601 & Strong \\
344 & 16,0294 & Strong \\
8 & 22,4848 & Strong \\
649 & 46,2663 & Strong \\
545 & 51,5411 & Strong \\
173 & 71,1571 & Strong \\
558 & 55,4951 & Strong \\
409 & 73,659 & Strong \\
371 & 74,0232 & Strong \\
\hline
\end{tabular}

MagMAX FFPE DNA/RNA Ultra kit (ThermoFisher Scientific Inc, Waltham, MA, USA). All procedures were carried out in accordance with the manufacturer's instructions. The extracted gDNA was quantified by Qubit 3 Fluorometer with Qubit dsDNA HS Assay Kit. The amount of amplifiable gDNA was determined by the TaqMan RNase P assay (ThermoFisher Scientific Inc).

Targeted exome sequencing. The targeted exome sequencing was performed on the Ion S5XL next-generation sequencing system with a custom designed Colorectal Cancer Gene Panel (ThermoFisher Scientific Inc) (Table II). The custom panel was designed to cover high mutation frequency in Colorectal Cancer from various genomic databases such as Catalogue Of Somatic Mutations in Cancer (COSMIC) database (https://cancer.sanger.ac.uk/cosmic/), cBioPortal (http://www.cbioportal.org/) and IntOGen (https://www.intogen.org). This panel contains 270 primer pairs in 3 pools and covers all coding regions of APC, TP53 and ARIDA1 genes, and hotspot region of 11 genes (AKT1, ATM, BRAF, CTNNB1, EGFR, FBXW7, KRAS, NRAS, PIK3CA, PTEN and SMAD4) (Table II). The amplicon library was constructed from 30 ng of amplifiable gDNA by Ion Ampliseq Library Plus Kit. The targeted areas were amplified by polymerase chain reaction for 20 cycles. The resulting amplicons were treated with FuPa reagent to partially digest primers. Amplicons were ligated to Ion P1 and Ion Xpress barcode adapters and purified using Agencourt AMPure XP reagent (Beckman Coulter). Barcoded libraries were quantified using the Ion Library TaqMan Quantitation Kit (ThermoFisher Scientific Inc) and diluted to a final concentration of $65 \mathrm{pM}$. The sequencing template preparation was done using Ion Chef with Ion 540 Chef Kits. Sequencing was performed for 500 flows on an Ion S5XL Sequencer with Ion 540 chip.
Table II. Ion Ampliseq Custom Colorectal Cancer Panel.

\begin{tabular}{lc}
\hline Gene & Coverage \\
\hline APC & $99.9 \%$ of full CDS \\
ARIDA1 & $96.2 \%$ of full CDS \\
TP53 & $100 \%$ of full CDS \\
AKT1 & Hotspots \\
ATM & Hotspots \\
BRAF & Hotspots \\
CTNNB1 & Hotspots \\
EGFR & Hotspots \\
FBXW7 & Hotspots \\
KRAS & Hotspots \\
NRAS & Hotspots \\
PIK3CA & Hotspots \\
PTEN & Hotspots \\
SMAD4 & Hotspots \\
\hline
\end{tabular}

CDS: Coding sequence.

Next generation sequencing data analysis. The Ion Torrent platformspecific pipeline software, Torrent Suite version 5.0.5 (Thermo Fisher Scientific Inc) was used to separate barcoded reads and to filter and remove poor signal reads. The coverage analysis was performed using the coverage analysis plug-in v5.0.4. BAM format files were generated from the sequencing results and then exported to the Ion Reporter Server (ThermoFisher Scientific Inc). The bioinformatics analysis of the sequencing was performed with Ion Torrent platform specific bioinformatics software, Ion Reporter version 5.6. The Ion Reporter Tumor-Normal workflow was used for detection of single nucleotide polymorphisms (SNPs), insertions and deletions in the tumor and normal samples that calculated the intersection of variants in the 2 samples. The workflow performs a statistical evaluation of the likelihood that the tumor allele is not present in the normal sample and calculates a $p$-value representing the statistical confidence of that call. The detected somatic mutations were verified in the Integrative genome viewer (IGV) from the Broad Institute (http://www.broadinstitute.org/igv/). The ion reporter workflow also annotates the variants with information from dbSNP, ExAC, 1,000 genomes, 5,000 exomes, OMIM, COSMIC, Polyphen, and ClinVar.

Sanger sequencing validation. Sanger sequencing-based validation was done on mutations in APC and PIK3CA genes detected by the custom designed Ion Ampliseq custom Colorectal Cancer Panel. The nucleotide sequences of the primers in the custom panel were obtained from ampliseq.com. The M13 forward primer sequence 5'TGTA AAACGACGG CCAGT-3' was added to the 5' end of the forward primer sequence, and the M13 reverse primer sequence 5, CAGGAAACAGCTATGACC-3' was added to the 5' end of the reverse primer sequence (Integrated DNA Technologies, Coralville, IA, USA). One ng of FFPE DNA was used for amplification of target region. Sanger sequencing was performed at The Centre for Applied Genomics (TCAG) at The Hospital for Sick Children, Toronto, Canada (Figure 2). The sequencing data was analyzed by FinchTV version 1.4.0 (Geospiza Inc.).

Microsatellite instability (MSI) analysis. Microsatellite instability status was assessed using two mononucleotide markers (BAT-25 and 

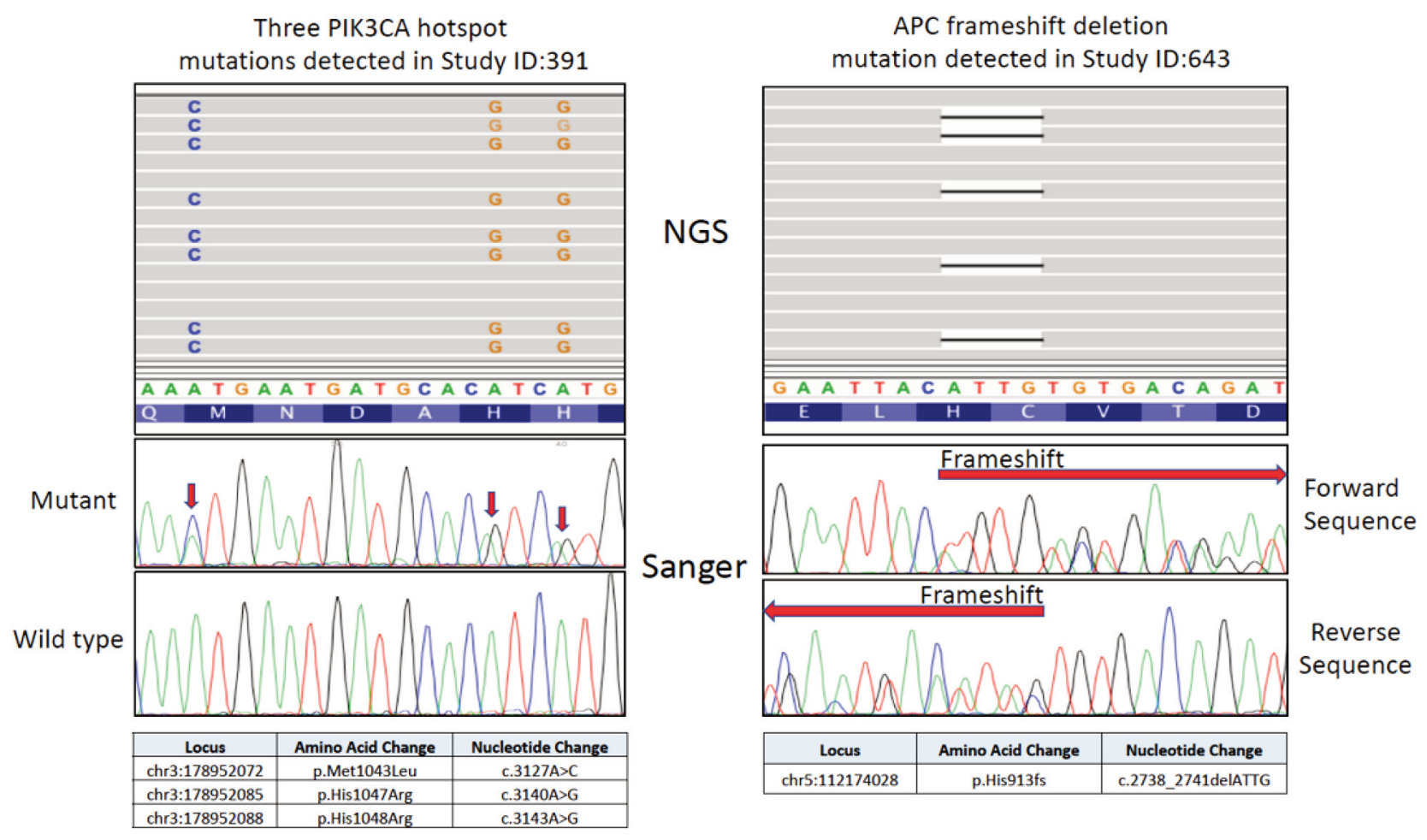

Figure 2. Sanger sequencing validation of PIK3CA (left panel) and adenomatosis polyposis coli (APC) mutations (right panel). Three PIK3CA mutations (Met1043Lue, His1047Arg and His1048Arg) in Study ID 391 and APC mutation (His913fs) in Study ID 643 were validated by Sanger Sequencing and compared with next generation sequencing (NGS) data. Upper panel shows NGS data viewed in the Integrative genome viewer, middle panel shows Sanger sequencing data viewed in the Finch TV software, lower panel shows Locus, amino acid changes and Nucleotide changes of the mutations.

BAT-26) and three dinucleotide markers (D5S346/APC, D2S123 and D17S250/MFD15) based on their high sensitivity as markers for cancer microsatellite instability status. Primers were fluorescently labelled and PCR amplified fragments were analyzed on an ABI 3500 XL Genetic Analyser using GeneMapper Software 5.0 (Applied Biosystems, Carlsbad, CA, USA) (Table III). The aggregate results of the 5 loci were compared between tumor and normal DNA and interpreted as microsatellite stable (MSS, 0 of 5 loci unstable) or low frequency microsatellite instability (MSI-L, 1 of 5 loci unstable) or high frequency microsatellite instability (MSI$\mathrm{H}, 2$ or more of 5 loci instable. A partial ( $>50 \%$ ) to complete signal loss of one heterozygote allele was indicated as $\mathrm{LOH}$ (loss of heterozygosity) (Table III). All procedures were performed at the molecular pathology laboratory at the Sunnybrook Health Sciences Centre.

Statistical analysis. The number of germline and somatic Adenomatosis Polyposis Coli (APC) mutations were compared between TTE groups using Kruskal-Wallis test. The number of patients in each group harboring 2 or more APC mutations, as well as other specific mutations detected in our custom panel (TP53 and ARID1A genes and hot spot regions of AKT1, ATM, BRAF, CTNNB1, EGFR, FBXW7, GNAS, KRAS, NRAS, PIK3CA, PTEN and SMAD4) were compared with Mann-Whitney and/or $t$-test depending on the distributions. $p$-Value $<0.05$ determined statistical significance.

\section{Results}

In this study, we carried out genomic profiling of tumors clustered by TTE, and investigated the association between TTE and identified mutations. CRCLM and normal liver from post-hepatectomy pathology specimens were used for next generation sequencing. For variant detection, the mean read depth of 8,693X (between 6,041 and 15,025X) for tumor tissue and 9,280X (between 5,628 and 14,368X) for adjacent normal tissue over the targeted genomic regions were achieved.

A total of 42 non-synonymous somatic mutations were detected from 10 patients with weak TTE (mean of 4.2 mutations) (Table IV) and a total of 26 non-synonymous somatic mutations were detected from 10 patients with strong TTE (mean of 2.6 mutations) (Table V) when compared to respective normal tissue samples. The mutation burden between weak TTE and strong TTE tumors was statistically different $(p=0.0137)$. APC was the most commonly altered gene, 30 mutations in 17 patients $(85 \%)$ followed by 11 KRAS mutations in 11 patients $(55 \%)$ and 10 TP53 mutations in 10 patients $(50 \%)$. There were no hotspot mutations detected in the CTNNB1 and ATM genes in this study. 
Table III. Magnetic resonance imaging (MRI) phenotype (weak versus strong target tumor enhancement) and microsatellite instability (MSI) status of each case.

\begin{tabular}{|c|c|c|c|c|c|c|c|}
\hline \multirow[t]{2}{*}{ MRI category } & \multirow[t]{2}{*}{ Study ID } & \multirow[t]{2}{*}{ MSI status } & \multicolumn{5}{|c|}{ MSI markers } \\
\hline & & & BAT-25 & BAT-26 & D5S346 & D2S123 & $\mathrm{D} 17 \mathrm{~S} 250$ \\
\hline \multirow[t]{10}{*}{ Weak TTE } & 249 & MSI-H & & & $\mathrm{LOH}$ & $\mathrm{LOH}$ & $\mathrm{LOH}$ \\
\hline & 15 & & & & & & \\
\hline & 659 & & & & & & \\
\hline & 292 & & & & & & \\
\hline & 568 & MSI-L & & & POS & & \\
\hline & 643 & & & & & & \\
\hline & 391 & MSI-H & & & & POS & POS \\
\hline & 778 & MSI-L & & & & POS & \\
\hline & 288 & & & & & & \\
\hline & 314 & & & & & & \\
\hline \multirow[t]{10}{*}{ Strong TTE } & 651 & & & & & & \\
\hline & 531 & MSI-L & & & POS & & \\
\hline & 344 & & & & & & \\
\hline & 8 & & & & & & \\
\hline & 649 & & & & & & \\
\hline & 545 & & & & & & \\
\hline & 173 & MSI-H & & & POS & POS & \\
\hline & 558 & & & & & & \\
\hline & 409 & MSI-L & & & & POS & \\
\hline & 371 & & & & & & \\
\hline
\end{tabular}

Among a total of 30 mutations detected in APC, 27 mutations $(90 \%)$ were either nonsense or frameshift mutations by insertion or deletion which are predicted to cause protein truncation or nonsense-mediated decay. Of 27 truncation mutations, 18 mutations $(66.6 \%)$ are found in the weak TTE and 9 mutations $(33.3 \%)$ in the strong TTE group. There was also a significant difference in the number of patients with double truncation mutations in APC between the weak TTE group $(8 / 10=80 \%)$ and the strong TTE group $(2 / 10=20 \%)$ using the Fisher's exact test $(p=0.01)$ Two cases in the weak TTE group carried single truncation APC mutations however allele mutation frequency of these mutations were relatively high at 76.6 and $71.3 \%$, respectively. All hotspot mutations in PIK3CA, PTEN, NRAS, BRAF, SMAD4, AKT1 and FBXW7 genes were only found in weak TTE group, however, the presence of KRAS hotspot mutations was not associated with either weak TTE $(6 / 10)$ or strong TTE group (5/10) (Tables IV and V).

In contrast, $80 \%$ of patients (8/10) with strong TTE tumors had TP53 mutations compared to $20 \%$ of patients (2/10) with weak TTE tumors. TP53 mutations in strong TTE were also strongly associated with APC mutations $(87.5 \%)$ (Tables IV and V). Microsatellite instability (MSI) analysis on the 10 weak TTE and the 10 strong TTE cases identified two MSI-H and two MSH-L cases in the weak TTE group compared to one MSI-H and two MSH-L cases in the strong TTE group. This result indicated that MSI status was not associated with either weak TTE or strong TTE. Interestingly, the two weak TTE cases which possessed a single APC truncated mutation at high mutation frequency (76.6 and $71.3 \%$ ) also carried microsatellite instability at APC loci (Table III).

\section{Discussion}

Our results show an association between TTE, mutation burden and mutation type in CRCLM. In our cohort, lesions with a low TTE had a higher mutation burden compared to lesions with high TTE. Similarly, some mutations were only present in the low-TTE group. In a previous study, it was shown that low TTE patients had a worst survival profile compared to high TTE patients, with a difference of around $30 \%$ at 3 years post hepatectomy (19). In this study, late gadolinium enhancement (as measured by TTE) was associated with histopathological evidence of tumour fibrosis. It is a well-known phenomenon that fibrosis in other disease processes demonstrate late enhancement on gadolinium enhanced MRI, including cardiac fibrosis following myocardial infarction or other cardiac diseases (20). Tumour fibrosis in CRCLM is a known histological predictor of long-term prognosis (21). It is hypothesized that this mechanism may explain why TTE is associated with 
Table IV. Somatic mutations detected in 10 patients with Weak target tumour enhancement (TTE) by Custom Colorectal Cancer Panel.

\begin{tabular}{|c|c|c|c|c|c|c|}
\hline Study ID & \# Locus & $\begin{array}{c}\text { Type } \\
\text { frequency }\end{array}$ & Mutation & Gene & Function & Protein \\
\hline
\end{tabular}

$(\%)$

\begin{tabular}{|c|c|c|c|c|c|c|c|}
\hline 15 & chr1:115256530 & SNV & 11,99 & NRAS & Missense & p.Gln61Lys & c. $181 \mathrm{C}>\mathrm{A}$ \\
\hline 314 & $\operatorname{chr} 1: 27023371$ & INDEL & 48,7 & ARID1A & Non-frameshift deletion & p.Ala160_Ala162del & c.478_486delGCCGTCGCC \\
\hline 778 & $\operatorname{chr} 1: 27101138$ & SNV & 33,3 & ARID1A & Nonsense & p.Gln1474Ter & c. $4420 \mathrm{C}>\mathrm{T}$ \\
\hline 288 & chr3:178936082 & SNV & 40,2 & PIK3CA & Missense & p.Glu542Lys & c. $1624 \mathrm{G}>\mathrm{A}$ \\
\hline 391 & chr3:178952072 & SNV & 68,9 & PIK3CA & Missense & p.Met1043Leu & c. $3127 \mathrm{~A}>\mathrm{C}$ \\
\hline 391 & chr3:178952085 & SNV & 65,2 & PIK3CA & Missense & p.His1047Arg & c. $3140 \mathrm{~A}>\mathrm{G}$ \\
\hline 314 & chr3:178952085 & SNV & 70 & PIK3CA & Missense & p.His 1047Arg & c. $3140 \mathrm{~A}>\mathrm{G}$ \\
\hline 391 & chr3:178952088 & SNV & 66,8 & PIK3CA & Missense & p.His 1048Arg & c. $3143 \mathrm{~A}>\mathrm{G}$ \\
\hline 659 & chr4:153247289 & SNV & 43,94 & FBXW7 & Missense & p.Arg505Cys & c. $1513 \mathrm{C}>\mathrm{T}$ \\
\hline 15 & chr5:112116592 & SNV & 44,91 & APC & Nonsense & p.Arg213Ter & c. $637 \mathrm{C}>\mathrm{T}$ \\
\hline 778 & chr5:112136976 & INDEL & 35,1 & APC & Frameshift deletion & p.Arg244fs & c.731_731delG \\
\hline 288 & chr5:112151204 & SNV & 16,9 & APC & Nonsense & p.Arg283Ter & c. $847 \mathrm{C}>\mathrm{T}$ \\
\hline 314 & chr5:112164586 & SNV & 15,7 & APC & Nonsense & p.Arg554Ter & c. $1660 \mathrm{C}>\mathrm{T}$ \\
\hline 292 & chr5:112170858 & SNV & 6,11 & APC & Missense & p.His652Tyr & c. $1954 \mathrm{C}>\mathrm{T}$ \\
\hline 778 & chr5:112173917 & SNV & 31,3 & APC & Nonsense & p.Arg876Ter & c. $2626 \mathrm{C}>\mathrm{T}$ \\
\hline 643 & chr5:112174028 & INDEL & 45,1 & APC & Frameshift deletion & p.His913fs & c.2738_2741delATTG \\
\hline 391 & chr5:112174096 & SNV & 19,9 & APC & Nonsense & p.Tyr935Ter & c. $2805 \mathrm{C}>\mathrm{A}$ \\
\hline 659 & chr5:112174357 & INDEL & 40,44 & APC & Frameshift insertion & p.Thr1023fs & c.3066_3067insA \\
\hline 292 & chr5:112174582 & INDEL & 24,74 & APC & Frameshift deletion & p.Val1099fs & c.3292_3293delTG \\
\hline 659 & chr5:112175211 & INDEL & 49,34 & APC & Frameshift deletion & p.Glu1309fs & c.3921_3925delAAAAG \\
\hline 292 & chr5:112175224 & INDEL & 24,86 & APC & Frameshift deletion & p.Gly1312fs & c.3934_3934delG \\
\hline 15 & chr5:112175348 & SNV & 46,01 & APC & Nonsense & p.Glu1353Ter & c. $4057 \mathrm{G}>\mathrm{T}$ \\
\hline 568 & chr5:112175390 & SNV & 71,29 & APC & Nonsense & p.Gln1367Ter & c. $4099 \mathrm{C}>\mathrm{T}$ \\
\hline 288 & chr5:112175575 & INDEL & 28,5 & APC & Frameshift deletion & p.Gln1429fs & c. $4285 \_4285 \mathrm{delC}$ \\
\hline 314 & chr5:112175591 & INDEL & 38 & APC & Frameshift deletion & p.Ser1434fs & c.4301_4301delG \\
\hline 249 & chr5:112175672 & INDEL & 76,59 & APC & Frameshift deletion & p.Ser1465fs & c.4385_4386delAG \\
\hline 643 & chr5:112175672 & INDEL & 41,4 & APC & Frameshift deletion & p.Ser1465fs & c.4385_4386delAG \\
\hline 391 & chr5:112175868 & INDEL & 43,4 & APC & Frameshift deletion & p.Pro1527fs & c.4578_4578delT \\
\hline 249 & chr7:140453136 & SNV & 35,94 & BRAF & Missense & p.Val600Glu & c. $1799 \mathrm{~T}>\mathrm{A}$ \\
\hline 15 & chr10:89624284 & SNV & 46,15 & PTEN & Missense & p.Gly20Arg & c. $.58 \mathrm{G}>\mathrm{A}$ \\
\hline 778 & chr10:89692899 & SNV & 43,3 & PTEN & Missense & p.Lys128Thr & c. $383 \mathrm{~A}>\mathrm{C}$ \\
\hline 391 & chr12:25378562 & SNV & 57,3 & KRAS & Missense & p.Ala146Thr & c. $436 \mathrm{G}>\mathrm{A}$ \\
\hline 288 & chr12:25398284 & SNV & 30 & KRAS & Missense & p.Gly 12 Val & c. $35 \mathrm{G}>\mathrm{T}$ \\
\hline 314 & $\operatorname{chr12:25398284}$ & SNV & 48,1 & KRAS & Missense & p.Gly12Val & c. $35 \mathrm{G}>\mathrm{T}$ \\
\hline 778 & chr12:25398284 & SNV & 59,8 & KRAS & Missense & p.Gly12Val & c. $35 \mathrm{G}>\mathrm{T}$ \\
\hline 643 & chr12:25398285 & SNV & 60,7 & KRAS & Missense & p.Gly $12 \mathrm{Arg}$ & c. $34 \mathrm{G}>\mathrm{C}$ \\
\hline 659 & chr12:25398285 & SNV & 59,73 & KRAS & Missense & p.Gly12Ser & c. $34 \mathrm{G}>\mathrm{A}$ \\
\hline 249 & chr14:105246527 & SNV & 3,94 & AKT1 & Missense & p.Arg25Cys & c. $73 \mathrm{C}>\mathrm{T}$ \\
\hline 249 & chr14:105246551 & SNV & 3,59 & AKT1 & Missense & p.Glu17Lys & c. $.49 \mathrm{G}>\mathrm{A}$ \\
\hline 314 & $\operatorname{chr17:7577123}$ & SNV & 45 & TP53 & Missense & p.Val272Glu & c. $815 \mathrm{~T}>\mathrm{A}$ \\
\hline 568 & chr17:7578476 & SNV & 60,67 & TP53 & Missense & p.Pro152Thr & c. $454 \mathrm{C}>\mathrm{A}$ \\
\hline 15 & chr18:48575163 & SNV & 85,65 & SMAD4 & Missense & p.Phe119Leu & c. $357 \mathrm{~T}>\mathrm{A}$ \\
\hline
\end{tabular}

survival. The association between TTE and survival was also shown to exist in both surgical and non-surgical CRCLM patients (22). In our cohort, most patients with weak TTE had two or more mutations in APC, whereas strong TTE lesions had most of the time no or single APC mutations.

Recently, APC mutations, initially thought to be ubiquitous amongst colorectal cancers, have received renewed interest as researchers realized that their numbers and type, as well as their associations with other mutations, were associated with outcomes in patients with colorectal cancer. For example, a recent study demonstrated that harboring two or more APC mutations was significantly associated with lower overall survival compared to none or one mutation (23) and another study showed that the double mutation of APC and PIK3CA negatively affected survival after hepatectomy (24). 
Table V. Somatic mutations detected in 10 patients with Strong target tumour enhancement (TTE) by the Custom Colorectal Cancer Panel.

\begin{tabular}{|c|c|c|c|c|c|c|c|}
\hline Study ID & \# Locus & $\begin{array}{c}\text { Type } \\
\text { frequency } \\
(\%)\end{array}$ & Mutation & Gene & Function & Protein & Coding \\
\hline 545 & $\operatorname{chr} 1: 27023402$ & SNV & 5,17 & ARID1A & Missense & p.His170Tyr & c. $.508 \mathrm{C}>\mathrm{T}$ \\
\hline 344 & chr1:27099390 & INDEL & 22,15 & ARID1A & Frameshift deletion & p.Gly1210fs & $\begin{array}{c}\text { c.3628_3641delGGG } \\
\text { TATCAGCCCAG }\end{array}$ \\
\hline 371 & chr5:112116592 & SNV & 5,01 & APC & Nonsense & p.Arg213Ter & c. $637 \mathrm{C}>\mathrm{T}$ \\
\hline 545 & chr5:112170852 & SNV & 14,81 & APC & Missense & p.Glu650Lys & c. $1948 \mathrm{G}>\mathrm{A}$ \\
\hline 558 & chr5:112173917 & SNV & 7,6 & APC & Nonsense & p.Arg876Ter & c. $2626 \mathrm{C}>\mathrm{T}$ \\
\hline 173 & chr5:112174631 & SNV & 55,68 & APC & Nonsense & p.Arg 1114Ter & c. $3340 \mathrm{C}>\mathrm{T}$ \\
\hline 545 & chr5:112174909 & INDEL & 47,14 & APC & Frameshift insertion & p.Thr1208fs & c.3618_3619insA \\
\hline 545 & chr5:112175211 & INDEL & 30,91 & APC & Frameshift deletion & p.Glu1309fs & c.3921_3925delAAAAG \\
\hline 344 & chr5:112175390 & SNV & 26,28 & APC & Nonsense & p.Gln1367Ter & c. $4099 \mathrm{C}>\mathrm{T}$ \\
\hline 173 & chr5:112175514 & SNV & 17,39 & APC & Missense & p.Glu1408Val & c. $4223 \mathrm{~A}>\mathrm{T}$ \\
\hline 651 & chr5:112175523 & INDEL & 64,1 & APC & Frameshift deletion & p.Ser1411fs & c. $4233 \_4233$ delT \\
\hline 558 & chr5:112175639 & SNV & 18,22 & APC & Nonsense & p.Arg1450Ter & c. $4348 \mathrm{C}>\mathrm{T}$ \\
\hline 409 & chr5:112175751 & INDEL & 35,09 & APC & Frameshift insertion & p.Leu1488fs & c.4460_4461insTT \\
\hline 651 & $\operatorname{chr} 12: 25378562$ & SNV & 73,4 & KRAS & Missense & p.Ala146Thr & c. $\overline{4} 36 \mathrm{G}>\mathrm{A}$ \\
\hline 558 & $\operatorname{chr12:25380275}$ & SNV & 15,36 & KRAS & Missense & p.Gln61His & c. $183 \mathrm{~A}>\mathrm{C}$ \\
\hline 409 & chr12:25398284 & SNV & 36,25 & KRAS & Missense & p.Gly12Val & c. $35 \mathrm{G}>\mathrm{T}$ \\
\hline 344 & $\operatorname{chr12:25398284}$ & SNV & 24,11 & KRAS & Missense & p.Gly12Asp & c. $35 \mathrm{G}>\mathrm{A}$ \\
\hline 649 & $\operatorname{chr} 12: 25398285$ & SNV & 17,4 & KRAS & Missense & p.Gly 12 Ser & c. $34 \mathrm{G}>\mathrm{A}$ \\
\hline 651 & chr17:7577094 & SNV & 49,7 & TP53 & Missense & p.Arg282Trp & c. $844 \mathrm{C}>\mathrm{T}$ \\
\hline 371 & $\operatorname{chr} 17: 7577117$ & SNV & 5,96 & TP53 & Missense & p.Val274Asp & c. $821 \mathrm{~T}>\mathrm{A}$ \\
\hline 173 & chr17:7577141 & SNV & 66,67 & TP53 & Missense & p.Gly266Val & c. $797 \mathrm{G}>\mathrm{T}$ \\
\hline 344 & $\operatorname{chr17:7577143~}$ & INDEL & 24,38 & TP53 & Non-frameshift deletion & p.Leu265del & c.792_794delACT \\
\hline 649 & $\operatorname{chr} 17: 7578212$ & SNV & 6,1 & TP53 & Nonsense & p.Arg213Ter & c. $637 \mathrm{C}>\mathrm{T}$ \\
\hline 558 & $\operatorname{chr} 17: 7578272$ & MNV & 20,61 & TP53 & Missense & $\begin{array}{l}\text { p.Gln192_His193 } \\
\text { delinsHisAsn }\end{array}$ & c.576_577delGCinsTA \\
\hline 409 & $\operatorname{chr17:7578412}$ & SNV & 62,6 & TP53 & Missense & p.Val173Glu & c. $518 \mathrm{~T}>\mathrm{A}$ \\
\hline 545 & $\operatorname{chr} 17: 7578461$ & SNV & 74,85 & TP53 & Missense & p.Val157Phe & c. $469 \mathrm{G}>\mathrm{T}$ \\
\hline 8 & No mutations & & & & & & \\
\hline 531 & No mutations & & & & & & \\
\hline
\end{tabular}

With the exception of KRAS mutations, found in both groups, hot spot mutations, known for their negative association with survival (8), were only found in weak TTE lesions. On the other hand, TP53 mutations, which seem to be associated with a better outcome $(8,9)$ were found mostly in strong TTE patients. MSI status was not associated with either TTE group in this sample. Based on these early results, it seems that the lesions with weak TTE were harboring mutations linked to poorer outcomes.

There are limitations to our pilot study. Firstly, the sample size was small, and analysis of a much larger sample would be necessary in order to confirm these results. Secondly, lesions were selected based on their TTE, and not randomly chosen in the cohort. Indeed, only lesions with clearly low TTE and clearly high TTE were included. This was decided in this preliminary study to ensure that lesions were falling into one of these two groups and not at the margin. Analysis of the entire cohort across all ranges of TTE would be interesting to better understand the relationship between TTE and mutational status. Next, no attempt was made to correlate TTE, mutations and survival in this cohort. This was done purposely, because the sample size used was very small and the results would have limited value. Analysis of larger cohorts will be necessary to further investigate the association between mutations, TTE and survival in these patients.

In conclusion, these early results suggest an association between TTE and mutational status of CRCLM, which may offer some clues as to why TTE is associated with overall survival in patients with CRCLM. Analysis in much larger cohorts of patients are needed to further investigate and validate these promising preliminary findings.

\section{Conflicts of Interest}

The Authors have no financial or personal conflicts of interest to disclose. 


\section{Authors' Contributions}

H.C. and L.M. designed the study, carried out magnetic resonance imaging (MRI) protocol and analysis, helped with writing the paper and statistical analysis of the data. E.H. qualitatively assessed tissue morphology including fibrosis, necrosis, and viable tumor cells on each representative slide and marked areas of tumors on the $\mathrm{H}$ and E slides. C.L. helped with study design and selection of cohort. Y.A. did the next-generation sequencing experiments, data analysis and wrote parts of the paper. A.S. provided the conceptual and technical guidance for NGS work, analyzed the data and revised it critically for important intellectual content.

\section{Acknowledgements}

We thank Ms. Galina Koultchitski for performing MSI procedures. We thank Mr. Vikrum Seth for supporting MRI imaging data retrieval. This study received funding through the Academic Health Science Centre Alternative Funding Plan (AHSC AFP) Innovation Fund and the Canadian Heads of Academic Radiology (CHAR) / GE Healthcare Development Award.

\section{References}

1 American Cancer Society. Cancer Facts \& Figures 2020. Available at: https://www.cancer.org/research/cancer-factsstatistics/all-cancer-facts-figures/cancer-facts-figures-2020.html [Last accessed on December 1, 2020]

2 Canadian Cancer Society. Colorectal Cancer Statistics 2019. Available at: https://www.cancer.ca/ /media/cancer.ca/CW/ cancer\%20information/cancer\%20101/Canadian\%20cancer\%20s tatistics/Canadian-Cancer-Statistics-2019-EN.pdf?la=en [Last accessed on December 1, 2020]

3 Kanas GP, Taylor A, Primrose JN, Langeberg WJ, Kelsh MA, Mowat FS, Alexander DD, Choti MA and Poston G: Survival after liver resection in metastatic colorectal cancer: review and meta-analysis of prognostic factors. Clin Epidemiol 4: 283-301, 2012. PMID: 23152705. DOI: 10.2147/CLEP.S34285

4 Egger ME, Cannon RM, Metzger TL, Nowacki M, Kelly L, Tatum C, Scoggins CR, Callender GG, McMasters KM and Martin RC 2nd: Assessment of chemotherapy response in colorectal liver metastases in patients undergoing hepatic resection and the correlation to pathologic residual viable tumor. J Am Coll Surg 216(4): 845-56; discussion 856-7, 2013. PMID: 23415549. DOI: 10.1016/j.jamcollsurg.2012.12.037

5 Nagashima I, Oka T, Hamada C, Naruse K, Osada T and Muto T: Histopathological prognostic factors influencing long-term prognosis after surgical resection for hepatic metastases from colorectal cancer. Am J Gastroenterol 94(3): 739-743, 1999. PMID: 10086660. DOI: 10.1111/j.1572-0241.1999.00945.x

6 Brunner SM, Kesselring R, Rubner C, Martin M, Jeiter T, Boerner T, Ruemmele P, Schlitt HJ and Fichtner-Feigl S: Prognosis according to histochemical analysis of liver metastases removed at liver resection. Br J Surg 101(13): 1681-1691, 2014. PMID: 25331841. DOI: 10.1002/bjs.9627

7 Van Cutsem E, Köhne CH, Láng I, Folprecht G, Nowacki MP, Cascinu S, Shchepotin I, Maurel J, Cunningham D, Tejpar S, Schlichting M, Zubel A, Celik I, Rougier P and Ciardiello F: Cetuximab plus irinotecan, fluorouracil, and leucovorin as first- line treatment for metastatic colorectal cancer: updated analysis of overall survival according to tumor KRAS and BRAF mutation status. J Clin Oncol 29(15): 2011-2019, 2011. PMID: 21502544. DOI: 10.1200/JCO.2010.33.5091

8 Løes IM, Immervoll H, Sorbye H, Angelsen JH, Horn A, Knappskog S and Lønning PE: Impact of KRAS, BRAF, PIK3CA, TP53 status and intraindividual mutation heterogeneity on outcome after liver resection for colorectal cancer metastases. Int J Cancer 139(3): 647-656, 2016. PMID: 26991344. DOI: 10.1002/ijc.30089

9 Pietrantonio F, Biondani P, Perrone F, Di Bartolomeo M, Pacifici M, Milione M, Melotti F, Maggi C, Montemurro G, Bossi I, Mariani L and de Braud F: TP53 mutations in advanced colorectal cancer: the dark side of the moon. Oncology 86(5-6): 289-294, 2014. PMID: 24924261. DOI: 10.1159/000360088

10 Jones OM, Rees M, John TG, Bygrave S and Plant G: Biopsy of resectable colorectal liver metastases causes tumour dissemination and adversely affects survival after liver resection. Br J Surg 92(9): 1165-1168, 2005. PMID: 15997444. DOI: $10.1002 /$ bjs. 4888

11 Vignot S, Lefebvre C, Frampton GM, Meurice G, Yelensky R, Palmer G, Capron F, Lazar V, Hannoun L, Miller VA, André F, Stephens PJ, Soria JC and Spano JP: Comparative analysis of primary tumour and matched metastases in colorectal cancer patients: evaluation of concordance between genomic and transcriptional profiles. Eur J Cancer 51(7): 791-799, 2015. PMID: 25797355. DOI: 10.1016/j.ejca.2015.02.012

12 Kogita A, Yoshioka Y, Sakai K, Togashi Y, Sogabe S, Nakai T, Okuno K and Nishio K: Inter- and intra-tumor profiling of multiregional colon cancer and metastasis. Biochem Biophys Res Commun 458(1): 52-56, 2015. PMID: 25623536. DOI: 10.1016/ j.bbrc.2015.01.064

13 Watanabe T, Kobunai T, Yamamoto Y, Matsuda K, Ishihara S, Nozawa K, Iinuma H, Shibuya H and Eshima K: Heterogeneity of KRAS status may explain the subset of discordant KRAS status between primary and metastatic colorectal cancer. Dis Colon Rectum 54(9): 1170-1178, 2011. PMID: 21825899. DOI: 10.1097/DCR.0b013e31821d37a3

14 Goranova TE, Ohue M, Shimoharu Y and Kato K: Dynamics of cancer cell subpopulations in primary and metastatic colorectal tumors. Clin Exp Metastasis 28(5): 427-435, 2011. PMID: 21380628. DOI: $10.1007 / \mathrm{s} 10585-011-9381-0$

15 Gerlinger M, Rowan AJ, Horswell S, Math M, Larkin J, Endesfelder D, Gronroos E, Martinez P, Matthews N, Stewart A, Tarpey P, Varela I, Phillimore B, Begum S, McDonald NQ, Butler A, Jones D, Raine K, Latimer C, Santos CR, Nohadani M, Eklund AC, Spencer-Dene B, Clark G, Pickering L, Stamp G, Gore M, Szallasi Z, Downward J, Futreal PA and Swanton C: Intratumor heterogeneity and branched evolution revealed by multiregion sequencing. N Engl J Med 366(10): 883-892, 2012. PMID: 22397650. DOI: 10.1056/NEJMoa1113205

16 Floriani I, Torri V, Rulli E, Garavaglia D, Compagnoni A, Salvolini L and Giovagnoni A: Performance of imaging modalities in diagnosis of liver metastases from colorectal cancer: a systematic review and meta-analysis. J Magn Reson Imaging 31(1): 19-31, 2010. PMID: 20027569. DOI: 10.1002/jmri.22010

17 Niekel MC, Bipat S and Stoker J: Diagnostic imaging of colorectal liver metastases with CT, MR imaging, FDG PET, and/or FDG PET/CT: a meta-analysis of prospective studies including patients who have not previously undergone treatment. 
Radiology 257(3): 674-684, 2010. PMID: 20829538. DOI: 10.1148/radiol.10100729

18 Cheung HMC, Karanicolas PJ, Coburn N, Seth V, Law C and Milot L: Delayed tumour enhancement on gadoxetate-enhanced MRI is associated with overall survival in patients with colorectal liver metastases. Eur Radiol 29(2): 1032-1038, 2019. PMID: 29992388. DOI: 10.1007/s00330-018-5618-5

19 Cheung HMC, Karanicolas PJ, Hsieh E, Coburn N, Maraj T, Kim JK, Elhakim H, Haider MA, Law C and Milot L: Late gadolinium enhancement of colorectal liver metastases postchemotherapy is associated with tumour fibrosis and overall survival post-hepatectomy. Eur Radiol 28(8): 3505-3512, 2018. PMID: 29476216. DOI: 10.1007/s00330-018-5331-4

20 Vogel-Claussen J, Rochitte CE, Wu KC, Kamel IR, Foo TK, Lima JA and Bluemke DA: Delayed enhancement MR imaging: utility in myocardial assessment. Radiographics 26(3): 795-810, 2006. PMID: 16702455 . DOI: $10.1148 / \mathrm{rg} .263055047$

21 Reddy SK, Parker RJ, Leach JW, Hill MJ and Burgart LJ: Tumor histopathology predicts outcomes after resection of colorectal cancer liver metastases treated with and without pre-operative chemotherapy. J Surg Oncol 113(4): 456-462, 2016. PMID: 27100028. DOI: $10.1002 /$ jso.24144
22 Cheung HMC, Kim JK, Hudson J, Coburn N, Karanicolas PJ, Law $\mathrm{C}$ and Milot L: Late gadolinium MRI enhancement of colorectal liver metastases is associated with overall survival among nonsurgical patients. Eur Radiol 29(7): 3901-3907, 2019. PMID: 30937587. DOI: 10.1007/s00330-019-06177-w

23 Schell MJ, Yang M, Teer JK, Lo FY, Madan A, Coppola D, Monteiro AN, Nebozhyn MV, Yue B, Loboda A, Bien-Willner GA, Greenawalt DM and Yeatman TJ: A multigene mutation classification of 468 colorectal cancers reveals a prognostic role for APC. Nat Commun 7: 11743, 2016. PMID: 27302369. DOI: 10.1038/ncomms 11743

24 Yamashita S, Chun YS, Kopetz SE, Maru D, Conrad C, Aloia TA and Vauthey JN: APC and PIK3CA mutational cooperativity predicts pathologic response and survival in patients undergoing resection for colorectal liver metastases. Ann Surg 272(6): 10801085, 2020. PMID: 28379870. DOI: 10.1097/SLA.00000000 00002245

Received May 16, 2021

Revised May 31, 2021

Accepted June 14, 2021 